\title{
Oversimplification of Dementia Risk Reduction Messaging Is a Threat to Knowledge Translation in Dementia Prevention Research
}

\author{
K.J. Anstey, R. Peters

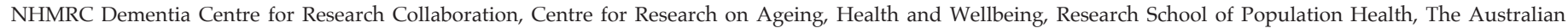 \\ National University, Australia.

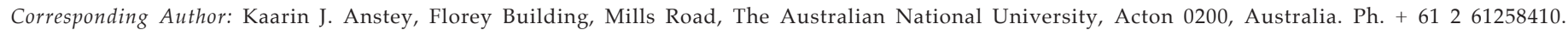 \\ kaarin.anstey@anu.edu.au \\ J Prev Alz Dis 2018;5(1):2-4 \\ Published online July 11, 2017, http:/ / dx.doi.org/10.14283/jpad.2017.27
}

7 The evidence for specific risk factors for Alzheimer's disease, vascular dementia and all cause dementia is increasing rapidly in quantity and quality. This has enabled the compilation of risk assessment tools for Alzheimer's disease (1), and their validation (2). It has also supported the promulgation of public health messaging about dementia risk reduction or dementia prevention. In general these developments are strong advances in the field of dementia prevention. However, the oversimplification of the findings and possible over-or mis-interpretation of their meaning, poses risks to accurate and effective knowledge translation in this field. Lack of balance in the interpretation of evidence on risk factors for dementia may lead to trials of interventions for dementia prevention that are ineffective. This will waste resources and create pessimism about dementia prevention research.

Two potential problems that may occur when translating evidence from observational research into prescriptions for prevention are identified here. The first is the generalisation of specific findings about risk factors either to multiple types of dementia or to multiple populations, when the evidence is in fact relevant to specific populations or outcomes. The second is the inference that reversal of a risk factor will lead to prevention, without knowledge of the threshold at which a factor becomes a 'risk', or evidence that reversal of the risk factor also reverses neuropathological processes instigated or caused by the risk factor.

\section{Generalisation of specific findings without good evidence}

Public awareness websites often say 'What's good for the heart is good for the brain'. Simple statements are needed for communicating public health messages to the wider public but there is a risk that oversimplification of messages will reduce the efficacy of prevention strategies and advice. Systematic review and observational studies have identified age-ranges at which cardiovascular risk factors appear to increase late-life dementia risk, and that results differ for Alzheimer's disease and Vascular dementia. Important subgroups could also include gender and presence of the APOE e4 genotype.

An example of generalisation of a specific finding to all of dementia occurs with hypertension. Many checklists and public information sites for dementia prevention advocate treatment of hypertension to reduce risk of dementia and imply that hypertension is a risk factor for dementia which most people would assume includes Alzheimer's disease (AD). However, epidemiological evidence currently does not support a clear link between blood pressure and pure AD (3). Older epidemiological studies also used less specific criteria for categorising dementia subtypes which impacts on the specificity of findings on risk factors and dementia sub-types. The World Alzheimer's report 2014 (4) states 'Overall, the more robust AlzRisk meta-analyses suggest no association between hypertension, systolic blood pressure or diastolic blood pressure and the incidence of $\mathrm{AD}^{\prime}$ (page 68).

Another example where there have been generalisations about evidence of a risk factor for dementia is in the case of high cholesterol where treating high cholesterol is part of dementia prevention strategies. Systematic review evidence has shown there is no consistent evidence linking high cholesterol in midlife or late-life to risk of vascular dementia, and no consistent evidence linking high cholesterol in late-life to risk of AD (5). Only midlife high cholesterol has been linked to latelife AD but the evidence for this is drawn from only two studies. If high cholesterol in late-life is not a risk factor for dementia, then trials of statins to prevention dementia are not indicated, and indeed, statins have not been shown to prevent dementia $(6,7)$.

\section{Assuming risk reversal leads to prevention without good knowledge of risk thresholds or identification of mechanisms}

Risk reduction interventions assume that modifying risk factors will lead to reduced rates of incident dementia. However, for this to occur, we need to know 
the level of exposure for each risk factor, age of exposure that increases risk, and the degree of reversal of the risk factor that reverses risk. Presently, in many areas we only have very general knowledge of associations, and lack specific information on the parameters at which risk and risk reduction can occur. An example of this is high blood pressure, arguably one of the most established risk factors. The evidence suggests that midlife may represent a critical period when exposure to high blood pressure is likely to increase risk of poorer cognition in late-life (8). However, evidence in latelife is mixed and may be confounded by falling blood pressure due to undiagnosed cognitive decline. There is now also emerging evidence for taking a life-course approach to exposure not least as the latter may take account of varying exposure levels over time (8-10). Our understanding of the level of blood pressure which confers risk is also evolving. This is inevitably confounded by the patterns of blood pressure lowering treatment, thresholds and targets in the varying epidemiological populations under study. For example; the level of blood pressure thought to be associated with an increased risk of cardiovascular events and thus meriting treatment has been falling steadily over the last 40 years (11).

We know even less about intervention to reverse exposure. Few trials of antihypertensive agents have included cognitive outcomes and each trial has, almost inevitably, focused on a differing population group and used differing treatment regimens, inclusion criteria, blood pressure targets and in general relatively short follow up (8). We know that blood pressure lowering reduces risk of cerebrovascular events and is therefore likely to protect cognitive function. We do not yet know at what level blood pressure becomes a cognitive risk factor, what exposure pattern or age is important and to what level we should lower blood pressure to protect the brain. A further complication related to pharmacological interventions is the possibility that differing trial outcomes may be influenced by their respective treatment regimens over and above any blood pressure lowering (12). This further underscores the need to fully understand the mechanisms of both the risk factor and the potential intervention.

In the field of non-pharmacological interventions, attempts to 'reverse' risk factors are often based on very little evidence about the target mechanism. For example, we do not know exactly why social engagement may reduce risk of dementia (13) or the conditions under which it is protective. Interventions to increase social engagement are therefore based on general principles rather than clear biological models. For cognitive activity, the evidence of protection has been gained from studies that measured activities such as reading, writing letters, using libraries, or having cognitively complex occupations (14). However, interventions to increase cognitive activity have often focused on brain-training
(15). These have not yet been shown to be robustly associated with reduced incident dementia. It has not yet been established how computerised brain training compares to lifestyle cognitive activity in reducing risk of dementia. It is possible that brain training, if effective, has a different mechanism than those other cognitively engaging activities measured in the questionnaires used in seminal studies on cognitive engagement and dementia risk.

Meta-analyses of the association between physical activity and dementia risk have pooled findings from studies using general physical activity questionnaires that summate all types of physical activities or exercise into global categories of type or level (e.g. aerobic or non-aerobic, high, medium and low). This is a result of the harmonization of data needed for meta-analyses (16). In contrast, intervention design requires a high degree of specificity. Fortunately neuroimaging studies provide evidence of neurobiological changes associated with physical activity $(17,18)$ and significant progress has been made in identifying putative mechanisms and associations between physical activity and neuropathology (19).

Overall, these arguments point to the great need for continued, rigorous research in the field of dementia prevention that takes care to specify exposures and outcomes and to calibrate effects and risk thresholds. Interventions need to be targeted appropriately and to those in whom there is scope to modify risk. Without very thorough, dementia-specific research into risk and protective factors, there is a danger of recommending preventive actions that are ineffective, that actually cause harm, or have an opportunity cost with no benefit. Smoking (20), anti-inflammatories (21), HRT, and statins have been thought to be risk reducing at various times as individual publications have shown protective effects. Over time, smoking has been shown to unequivocally increase risk, and there is inconclusive evidence for the other three factors, with some studies even showing an increased risk in some populations associated with these medications.

Unlike prevention research in the field of diabetes or falls, research into dementia prevention is still relatively new, and the volume of studies is relatively small. However, expectations and hope for this field are very high. We need to communicate that patience is needed while we work out how to reverse risk factors or apply treatments that will be effective. If this painstaking research is not done, potential risk reduction strategies may be prematurely dismissed as ineffective in trials where the intervention has not been of adequate type, duration, or intensity or has not been targeted to the relevant population.

Conflict of interest: None. 


\section{References}

1. Barnes DE, Covinsky KE, Whitmer RA, Kuller LH, Lopez OL, Yaffe K. Predicting risk of dementia in older adults: The late-life dementia risk index. Neurology. 2009;73(3):173-9.

2. Anstey KJ, Cherbuin N, Herath P, Qui C, Kuller LH, Lopez OL, et al. A self report risk index to predict occurence of dementia in three independent cohorts of older adults: The ANU-ADRI. PLoS One. 2014;9(1):e86141.

3. Power MC, Weuve J, Gagne JJ, McQueen MB, Viswanathan A, Blacker D. The association between blood pressure and incident Alzheimer disease: a systematic review and meta-analysis. Epidemiology. 2011;22(5):646-59.

4. Prince M, Albanese E, Guerchet M, Prina M. World Alzheimer's Report: Dementia and Risk Reduction, An analysis of protective and modifiable factors. London: Alzheimer's Disease International; 2014.

5. Anstey KJ, Ashby-Mitchell K, Peters R. Updating the Evidence on the Association between Serum Cholesterol and Risk of Late-Life Dementia: Review and Meta-Analysis. J Alzheimers Dis. 2017;56(1):215-28.

6. Power MC, Weuve J, Sharrett AR, Blacker D, Gottesman RF. Statins, cognition, and dementia-systematic review and methodological commentary. Nat Rev Neurol. 2015;11(4):220-9.

7. Shepherd J, Blauw GJ, Murphy MB, Bollen EL, Buckley BM, Cobbe SM, et al. Pravastatin in elderly individuals at risk of vascular disease (PROSPER): a randomised controlled trial. Lancet. 2002;360(9346):1623-30.

8. Iadecola C, Yaffe K, Biller J, Bratzke LC, Faraci FM, Gorelick PB, et al. Impact of Hypertension on Cognitive Function: A Scientific Statement From the American Heart Association. Hypertension. 2016;68(6):e67-e94.

9. Power MC, Schneider AL, Wruck L, Griswold M, Coker LH, Alonso A, et al. Life-course blood pressure in relation to brain volumes. Alzheimers Dement. 2016;12(8):890-9.

10. Power MC, Tchetgen EJ, Sparrow D, Schwartz J, Weisskopf MG. Blood pressure and cognition: factors that may account for their inconsistent association. Epidemiology. 2013;24(6):886-93.

11. Saklayen MG, Deshpande NV. Timeline of History of Hypertension Treatment. Front Cardiovasc Med. 2016;3:3.
12. Yasar S, Schuchman M, Peters J, Anstey KJ, Carlson MC, Peters R. Relationship Between Antihypertensive Medications and Cognitive Impairment: Part I. Review of Human Studies and Clinical Trials. Curr Hypertens Rep. 2016;18(8):67.

13. Fratiglioni L, Paillard-Borg S, Winblad B. An active and socially integrated lifestyle in late life might protect against dementia. Lancet Neurol. 2004;3(6):343-53.

14. Wilson RS, Scherr PA, Schneider JA, Tang Y, Bennett DA. Relation of cognitive activity to risk of developing Alzheimer disease. Neurology. 2007;69(20):1911-20.

15. Unverzagt FW, Guey LT, Jones RN, Marsiske M, King JW, Wadley VG, et al. ACTIVE cognitive training and rates of incident dementia. J Int Neuropsychol Soc. 2012;18(4):669-77.

16. Guure CB, Ibrahim NA, Adam MB, Said SM. Impact of Physical Activity on Cognitive Decline, Dementia, and Its Subtypes: Meta-Analysis of Prospective Studies. Biomed Res Int. 2017;2017:9016924.

17. Varma VR, Chuang Y, Harris GC, Tan EJ, Carlson MC. Low-intensity daily walking activity is associated with hippocampal volume in older adults. Hippocampus. 2014.

18. Erickson KI, Voss MW, Prakash RS, Basak C, Szabo A, Chaddock L, et al. Exercise training increases size of hippocampus and improves memory. Proc Natl Acad Sci U S A. 2011;108(7):3017-22.

19. Gallaway PJ, Miyake H, Buchowski MS, Shimada M, Yoshitake Y, Kim AS, et al. Physical Activity: A Viable Way to Reduce the Risks of Mild Cognitive Impairment, Alzheimer's Disease, and Vascular Dementia in Older Adults. Brain Sci. 2017;7(2)

20. Whitehouse PJ, Kalaria RN. Nicotinic receptors and neurodegenerative dementing diseases: basic research and clinical implications. Alzheimer Dis Assoc Disord. 1995;9 Suppl 2:3-5.

21. Szekely CA, Breitner JC, Fitzpatrick AL, Rea TD, Psaty BM, Kuller LH, et al. NSAID use and dementia risk in the Cardiovascular Health Study: role of APOE and NSAID type. Neurology. 2008;70(1):17-24. 